\title{
Some Aspects of Normal Quantisation
}

\author{
By
}

\author{
Keith C. HANNABUSS* and Mark A. Hennings**
}

\begin{abstract}
A generalisation of the process of normal quantisation is studied which deals with a physical system whose phase space is given by a coadjoint orbit of a locally compact Lie group G. Under certain conditions, an algebra structure-implemented by a Fronsdal *-product-is assigned to the family of physical observables in such a way that the normal quantisation provides a representation of the algebra. This method, then, provides a useful means of determining a physically sensible Fronsdal *-product for a given system.
\end{abstract}

\section{§. Introduction}

It is well-known that the traditional formalism of classical mechanics, where the phase space for the physical system is described by a symplectic manifold $(M, \omega)$ and the observables $\mathscr{A}$ form a Lie subalgebra of $C^{\infty}(M)$ under the Poisson bracket $\{$,$\} , is not one which can$ be conveniently reconciled to quantum mechanics. Indeed Moyal [10] has shown in the case $M=\mathbb{R}^{2 n}$ that, in order for classical mechanics and quantum mechanics to be correctly related, the Lie algebra structure $\{$,$\} of \mathscr{A}$ should be deformed to a new structure $\{*\}$ (the Moyal bracket), which is related to the Poisson bracket $\{$, in the sense that $\{f * g\}=\{f, g\}+O\left(\hbar^{2}\right)$ for $f, g \in \mathscr{A}$ and $\{f * g\}=\{f, g\}$ for $f \in \mathscr{A}$ and $g \in \mathscr{A}_{0}$, where $\mathscr{A}_{0}$ is the Lie subalgebra of $C^{\infty}(M)$ spanned by the constant functions and the $2 n$ coordinate functions $\xi_{1}, \ldots, \xi_{2 n}$ of $\mathbb{R}^{2 n}$. Indeed it has been shown that the algebra structure of pointwise multiplication on $C^{\infty}(M)$ can be deformed to a new associative structure * (the Moyal product) on $C^{\infty}(M)$ such that $f * g=$ $f \cdot g+O(\hbar)$ and $f * g-g * f=i \hbar\{f * g\}$ for all $f, g \in \mathscr{A}$.

Communicated by H. Araki, July 30, 1985 .

* Balliol College, Oxford OX1 3BJ, England.

** St. John's College, Oxford OX1 3JP, England. 
It has been suggested that this process of deformation is the correct approach towards quantising a physical system. Fronsdal [2] has proposed that, for a general physical system with phase space $(M, \omega)$, the algebra structure of the space $\mathscr{A}$ of observables should be deformed from pointwise multiplication to an associative structure * such that

$$
\begin{array}{ll}
f * g=f \cdot g+O(\hbar) & f, g \in \mathscr{A} ; \\
f * g-g * f=i \hbar\{f, g\} & f \in \mathscr{A}_{\circ}, g \in \mathscr{A},
\end{array}
$$

where $\mathscr{A}_{\circ}$ is the Lie subalgebra of $C^{\infty}(M)$ contained in $\mathscr{A}$ chosen to represent the basic geometrical symmetries of the system. Any such structure $*$ will be called an $\mathscr{A}_{\circ}$-invariant Fronsdal $*$-product on $\mathscr{A}$. Quantisation of the classical system is now to be achieved by finding a representation of the algebra $(\mathscr{A}, *)$.

Of course, the problem remains of how to choose a physically sensible Fronsdal *-product. In this paper we shall investigate a method which, under certain circumstances, generates meaningful Fronsdal *-products. The material in this paper relies partly on unpublished lecture notes of the first author [4], and partly on the D. Phil. thesis of the second author [5].

\section{§2. Motivation for the Construction}

Let $U$ be a projective unitary representation of a separable locally compact group $G$ with multiplier $\sigma$ on a Hilbert space $\mathscr{H}$ which has a unit cyclic vector $\Omega$. To specify our convention, this means that $U(g h)=\sigma(g, h) \cdot U(g) U(h)$ for all $g, h \in G$. The map taking a linear operator $A$ on $\mathscr{H}$ to the function

$$
\phi_{A}(g)=\langle U(g) \Omega, A U(g) \Omega\rangle \quad g \in G
$$

is well-known, as is its transpose which takes a probability measure $\mu$ to a quasi-classical density operator

$$
\rho_{\mu}=\int_{G}|U(g) \Omega\rangle\langle U(g) \Omega| d \mu(g) .
$$

If $G$ is a Lie group and $\Omega$ an analytic vector then the function $\Phi(g)$ $=\langle\Omega, U(g) \Omega\rangle$ can be differentiated to give

$$
f(X)=\left.i \hbar \frac{d}{d t} \Phi\left(e^{t X}\right)\right|_{t=0} \quad X \in \mathscr{G}
$$


so that $f \in \mathscr{G}^{*}$, the dual of the real Lie algebra $\mathscr{G}$ of $G$. Geometric quantisation theory tells us that the $G$-orbit of $f$ is a symplectic manifold $(M, \omega)$. If the stabiliser of $\Phi$ coincides with the stabiliser of $f$, then $\phi_{A}$ can be seen as a function on $M$. This provides a way of associating functions on a symplectic manifold with operators on a Hilbert space.

When $G$ is the additive group of a symplectic space, and the multiplier $\sigma$ is the exponential of the symplectic form, then the map taking $A$ to $\phi_{A}$ can be inverted to give the normal quantisation (see $\S 4)$. Consequently we shall call any linear map $N$ from functions on $M$ to operators on $\mathscr{H}$ such that

$$
\langle U(g) \Omega, N(\phi) U(g) \Omega\rangle=\phi(g \cdot f) \quad g \in G
$$

a generalised normal quantisation.

\section{§3. Notation and Elementary Results}

When considering a multiplier $\sigma$ on the group $G$, it is natural to look at the new function

$$
\tilde{\sigma}(g, h)=\sigma(g, h) \sigma\left(h, h^{-1} g h\right)^{-1} \quad g, h \in G
$$

(Hannabuss [3], Kleppner [9]). It is well-known that, if $G$ is abelian, then $\tilde{\sigma}$ is an antisymmetric bicharacter of $G$ which depends only on the equivalence class of $\sigma$. In the general case this is no longer true, but we shall now state the results concerning $\tilde{\sigma}$ that will interest us.

Proposition 1.

$$
\begin{array}{lll}
\text { (a) } \tilde{\boldsymbol{\sigma}}(g h, k)=\tilde{\boldsymbol{\sigma}}(g, h) \tilde{\boldsymbol{\sigma}}\left(h^{-1} g h, k\right) & g, h, k \in G \text {; } \\
\text { (b) } \tilde{\boldsymbol{\sigma}}\left(g, h^{-1}\right)^{-1}=\tilde{\boldsymbol{\sigma}}\left(h g h^{-1}, h\right) & g, h \in G ; \\
\text { (c) } U\left(h^{-1} g h\right)=\tilde{\boldsymbol{\sigma}}(g, h) U(h)^{*} U(g) U(h) g, h \in G .
\end{array}
$$

Proof. Straightforward, see Hannabuss [3].

If we introduce the spherical function $\Phi(g)=\langle\Omega, U(g) \Omega\rangle$, then formula (c) shows that

$$
\langle U(g) \Omega, U(k) U(g) \Omega\rangle=\tilde{\boldsymbol{\sigma}}(k, g)^{-1} \Phi\left(g^{-1} k g\right)
$$

for $g, k \in G$. In other words when the cyclic vector changes from $\Omega$ to $U(g) \Omega$ the spherical function changes to 


$$
(g \bullet \Phi)(k)=\tilde{\sigma}(k, g)^{-1} \Phi\left(g^{-1} k g\right) .
$$

One readily checks that this formula defines an action of $G$ on spherical functions, and we let $H_{1}$ be the stabiliser of $\Phi$, that is

$$
H_{1}=\left\{h \in G \mid \Phi\left(h^{-1} k h\right)=\tilde{\sigma}(k, h) \Phi(k) \quad \forall k \in G\right\} .
$$

We also introduce the subgroup $H_{2}$ which stabilises the ray through $\Omega$, that is

$$
H_{2}=\{h \in G \mid U(h) \Omega \in C \cdot \Omega\} .
$$

Proposition 2. $H_{2}$ can be characterised as $\{h \in G|| \Phi(h) \mid=1\}$, and it is a subgroup of $H_{1}$.

Proof. The condition for equality in the Cauchy-Schwarz inequality says that $|\Phi(h)|=|\langle\Omega, U(h) \Omega\rangle|=|| \Omega|| \cdot|| U(h) \Omega \|=1$ if and only if $U(h) \Omega$ is a multiple of $\Omega$, namely if and only if $h$ belongs to $H_{2}$. We also note that when $h \in H_{2}, U(h) \Omega=\langle\Omega, U(h) \Omega\rangle \Omega=\Phi(h) \Omega$, so that $\Phi$ is a unitary $\sigma$-character of $H_{2}$. Finally, if $h \in H_{2}$, then $(h \cdot \Phi)(k)$ $=\langle U(h) \Omega, U(k) U(h) \Omega\rangle=|\Phi(h)|^{2}\langle\Omega, U(k) \Omega\rangle=\Phi(k)$, so that $h \in H_{1}$.

Proposition 3. $\langle U(g h) \Omega, U(k) U(g h) \Omega\rangle=\langle U(g) \Omega, U(k) U(g) \Omega\rangle$ for $g, k \in G$ and $h \in H_{1}$.

Proof. $\langle U(g h) \Omega, U(k) U(g h) \Omega\rangle=[(g h) \cdot \Phi](k)=(g \circ \Phi)(k)=\langle U(g) \Omega$, $U(k) U(g) \Omega\rangle$.

Theorem 1. If $U$ is an irreducible $\sigma$-representation then $H_{1}=H_{2}$, so that we may drop the suffix and write $H=H_{1}=H_{2}$. For each bounded linear operator $A$ on $\mathscr{H}$ the function $\phi_{A}$ defined in (1) may be regarded as a function on $G / H$, with

$$
\phi_{A}(g H)=\langle U(g) \Omega, A U(g) \Omega\rangle
$$

for $g \in G$.

Proof. Since $U$ is irreducible the von Neumann algebra generated by the operators $U(k)$ is equal to the whole algebra $\mathscr{B}(\mathscr{H})$ of bounded linear operators on $\mathscr{H}$. Hence taking the weak operator limit of the result of Proposition 3 tells us that $\langle U(g h) \Omega, A U(g h) \Omega\rangle=\langle U(g) \Omega$, $A U(g) \Omega\rangle$ for all $g \in G, h \in H_{1}$ and $A \in \mathscr{B}(\mathscr{H})$. Taking $A=|\Omega\rangle\langle\Omega|$ and 
$g=1$, we deduce that $|\Phi(h)|^{2}=\|\Omega\| \|^{4}=1$ for all $h \in H_{1}$. It follows that $H_{1} \leqq H_{2}$, which combined with Proposition 1 yields $H_{1}=H_{2}$. The rest of the theorem now follows.

Remark. We shall henceforth assume that $U$ is irreducible.

Let us now also assume that $G$ is a Lie group, that $\Omega$ is an analytic vector, and that the multiplier $\sigma$ is also analytic. $\Phi$ may now be differentiated to obtain $f$ in $\mathscr{G}^{*}$ as in (3). It is natural to consider the $\sigma$-coadjoint action of $G$ on $\mathscr{G}^{*}$ defined by

$$
(g \bullet \phi)(X)=\phi\left(g^{-1} \cdot X\right)-i \hbar \tilde{\sigma}_{*}(X, g) \quad g \in G, \phi \in \mathscr{G} *, X \in \mathscr{G},
$$

where $g^{-1} \cdot X$ denotes the usual adjoint action of $G$ on $\mathscr{G}$, and $\tilde{\sigma}_{*}$ : $\mathscr{G} \times G \rightarrow \mathbb{C}$ is given by

$$
\tilde{\sigma}_{*}(X, g)=\left.\frac{d}{d t} \tilde{\sigma}\left(e^{t X}, g\right)\right|_{t=0} \quad X \in \mathscr{G}, g \in G
$$

(Hannabuss [3]). Let $K$ be the stabiliser of $f$ under the $\sigma$-coadjoint action.

\section{Proposition 4. $H \leqq K$.}

Proof. If $\xi$ is an analytic vector we can define $U_{*}(X) \xi$ for $X \in \mathscr{G}$ by $U_{*}(X) \xi=\left.\frac{d}{d t} U\left(e^{t X}\right) \xi\right|_{t=0}$. Then the definition of $f$ amounts to $f(X)=i \hbar\left\langle\Omega, U_{*}(X) \Omega\right\rangle$, and $(h \cdot f)(X)=i \hbar\left\langle U(h) \Omega, U_{*}(X) U(h) \Omega\right\rangle$ for any $h \in H$. It is now obvious that if $h$ is in $H$ it stabilises $f$, and the result follows.

If we let $M_{f}$ be the $\sigma$-coadjoint orbit of $f$, then geometric quantisation theory (Kirillov [8], Woodhouse [14]) tells us that $M_{f}$ is a symplectic manifold. Since any function on $C^{\infty}\left(M_{f}\right)$ may be regarded as a function on $G / K$, it follows that when $H=K$ we can use (1) and (10) to define a function $\phi_{A}$ in $C^{\infty}\left(M_{f}\right)$ for any $A \in \mathscr{B}(\mathscr{H})$ given by

$$
\phi_{A}(g \circ f)=\langle U(g) \Omega, A U(g) \Omega\rangle \quad g \in G 。
$$

When $H=K$, then there exists a $\sigma$-character on $K$ which differentiates to $f$, namely $\Phi$, and so geometric quantisation theory now tells us that the symplectic manifold $\left(M_{f}, \omega\right)$ is quantisable. When the map $A \mapsto \phi_{A}$ is injective, we may invert it to obtain a generalised 
normal quantisation.

Remark. It is by no means always the case that $H=K$, as the following example shows.

Example 1. Let $U$ be the natural real representation of $S O(n)$ on $\boldsymbol{R}^{n}$, and let $\Omega$ be any unit vector in $\boldsymbol{R}^{n}$. Since $U$ is unitary,

$$
\left\langle\Omega, U_{*}(X) \Omega\right\rangle=-\left\langle U_{*}(X) \Omega, \Omega\right\rangle=-\left\langle\Omega, U_{*}(X) \Omega\right\rangle \quad X \in \mathscr{G},
$$

so that $f(X)=i \hbar\left\langle\Omega, U_{*}(X) \Omega\right\rangle=0$, and since $\sigma \equiv 1$ we see that $K=G$. On the other hand, the stabiliser $H$ of the ray through $\Omega$ is isomorphic to $O(n-1)$ which is strictly smaller. By complexifying $U$ we could obtain the analogous result for a complex representation.

There are, however, some interesting examples in which both $H=K$ and the map $A \mapsto \phi_{A}$ is injective, and these form the subject of the next two sections.

\section{§4. The Group $R^{2 n}$}

For $x_{1}, x_{2}, y_{1}, y_{2}$ in $\boldsymbol{R}^{n}, \lambda\left(\left(x_{1}, x_{2}\right),\left(y_{1}, y_{2}\right)\right)=x_{1} \cdot y_{2}-x_{2} \cdot y_{1}$ defines a symplectic form on $\boldsymbol{R}^{2 n}$. Correspondingly $\sigma=\exp \frac{i \lambda}{2 \hbar}$ defines a multiplier on the additive group $G$ of $\boldsymbol{R}^{2 n}$. By the Stone-von Neumann theorem, every unitary irreducible $\sigma$-representation of $G$ is equivalent to that defined on $L^{2}\left(\mathbb{R}^{n}\right)$ by

$$
\left[U\left(x_{1}, x_{2}\right) \psi\right](q)=\exp \left(\frac{i}{\hbar} x_{2} \cdot\left(q-\frac{1}{2} x_{1}\right)\right) \cdot \psi\left(q-x_{1}\right) \quad \phi \in L^{2}\left(\boldsymbol{R}^{n}\right) .
$$

Consider the analytic vector $\Omega \in L^{2}\left(\boldsymbol{R}^{n}\right)$ given by

$$
\Omega(q)=\left(\frac{2}{h}\right)^{\frac{1}{t n}} \exp \left(-|q|^{2} / 2 \hbar\right) \quad q \in \mathrm{R}^{n}
$$

(here $|q|^{2}=q \cdot q$ ). A simple calculation shows that

$$
\Phi(x)=\exp \left(-|x|^{2} / 4 \hbar\right) \quad x \in \boldsymbol{R}^{2 n} \text {. }
$$

In this case $\mathscr{G}$ may be identified with $\boldsymbol{R}^{2 n}$ so that the exponential map is the natural identification of $\mathscr{G}$ with $G$, and so 


$$
f(X)=\left.i \hbar \frac{d}{d t} \exp \left(-t^{2}|X|^{2} / 4 \hbar\right)\right|_{t=0}=0 \quad X \in \mathscr{G}
$$

Also $\tilde{\sigma}_{*}(X, y)=\left.\frac{d}{d t} \exp \left(\frac{i t}{\hbar} \lambda(X, y)\right)\right|_{t=0}=\frac{i}{\hbar} \lambda(x, y)$ for $\quad X \in \mathscr{G}, y \in \mathscr{G}$, so that under the $\sigma$-coadjoint action

$$
(y \cdot f)(X)=\lambda(X, y) \quad X \in \mathscr{G}, y \in G .
$$

The fact that $\sigma$ is nondegenerate now forces $K=\{0\}$, and so by Proposition $4 H=\{0\}$ also. Consequently we deduce that $M_{f} \equiv \mathbb{R}^{2 n}$, and the methods of $\S 3$ lead us to look for functions on $\mathbb{R}^{2 n}$ of the form

$$
\phi_{A}(x)=\langle U(x) \Omega, A U(x) \Omega\rangle \quad A \in \mathscr{B}\left(L^{2}\left(\mathbb{R}^{n}\right)\right) .
$$

Since $\sigma$ is a type I multiplier on $\mathbb{R}^{2 n}$, there exists a natural Fourier transform $\mathscr{F}_{\sigma}$ given by

$$
\left(\mathscr{F}_{\sigma} \phi\right)(x)=h^{-n} \int_{G} \phi(y) \tilde{\sigma}(x, y) d y \quad \phi \in L(G)=L\left(\mathbb{R}^{2 n}\right) .
$$

It is known that $\mathscr{F}_{\sigma}$ maps Schwartz functions to Schwartz functions, and that $\mathscr{F}_{\sigma}^{2}=I$ (Reiter [12]). The Weyl quantisation $W$ of a function $\phi$ on $\boldsymbol{R}^{2 n}$ for which $\mathscr{F}_{\sigma} \phi \in L(G)$ is

$$
\left.\mathrm{W}(\phi)=U\left(\mathscr{F}_{\sigma} \phi\right)=h^{-n} \int_{G} U(x) \mathscr{F}_{\sigma} \phi\right)(x) d x .
$$

Now Hudson [6] has shown that any $G$-invariant quantisation must take $\phi$ to $U\left(F \cdot \mathscr{F}_{\sigma} \phi\right)$ for some function $F$. The significance of this is that $W$ is the transpose of the map taking a density operator $\rho$ to its Wigner probability measure

$$
\left(W^{\prime} \rho\right)(x)=\int_{G} \operatorname{tr}\left[\rho U(y)^{*}\right] \tilde{\sigma}(x, y) d \nu .
$$

The intrusion of $F$ has the effect of conditioning $W^{\prime} \rho$ by convoluting it with $\mathscr{F}_{\sigma} F$. Such convolutions occur, for instance, in the Husimi transform (Davies [1], Husimi [7]). We have observed that $\Phi$ is nonzero, and that particular $G$-invariant quantisation called normal quantisation is precisely that obtained by putting $F=\Phi^{-1}$.

Theorem 2. Let $\mathscr{S}$ be the linear space of Schwartz functions on $\mathbb{R}^{2 n}$ such that $\Phi^{-1} \cdot \mathscr{F}_{\sigma} \phi \in L\left(\mathbb{R}^{2 n}\right)$. A linear map $N$ from $\mathscr{S}$ to Hilbert-Schmidt operators on $L^{2}\left(\boldsymbol{R}^{n}\right)$ is a generalised normal quantisation if and only if $N(\phi)=U\left(\Phi^{-1} \cdot \mathscr{F}_{\sigma} \phi\right)$, i.e. if and only if $N$ is the normal quantisation. 
Proof. If $F \in L\left(\mathbb{R}^{2 n}\right)$ then

$$
\begin{aligned}
\langle U(x) \Omega, U(F) U(x) \Omega\rangle & =h^{-n} \int_{G} F(y)\langle U(x) \Omega, U(y) U(x) \Omega\rangle d y \\
& =h^{-n} \int_{G} F(y) \tilde{\sigma}(y, x)^{-1} \Phi(y) d y \\
& =\left[\mathscr{F}_{\sigma}(F \cdot \Phi)\right](x) .
\end{aligned}
$$

If $\phi \in \mathscr{S}$, then $\phi=\mathscr{F}_{\sigma}(F \cdot \Phi)$ for some $F \in L(G)$, and so $\langle U(x) \Omega, U$ $\left.\left(\Phi^{-1} \cdot \mathscr{F}_{\sigma} \phi\right) U(x) \Omega\right\rangle=\phi(x)$. Thus the map $N: \phi \mapsto U\left(\Phi^{-1} \cdot \mathscr{F}_{\sigma} \phi\right)$ is a generalised normal quantisation.

Conversely, suppose that $N$ is a generalised normal quantisation. Then for $\phi \in \mathscr{S}$ the Plancherel theorem states that $N(\phi)=U\left(F_{\phi}\right)$ for some $F_{\phi} \in L(G)$, and so the above argument tells us that $\phi(x)=$ $\langle U(x) \Omega, N(\phi) U(x) \Omega\rangle=\left[\mathscr{F}_{\sigma}\left(F_{\phi} \cdot \Phi\right)\right](x)$. Hence we deduce that $F_{\phi}=$ $\Phi^{-1} \cdot \mathscr{F}_{\sigma} \phi$ as required.

Thus we see that the map $A \mapsto \phi_{A}$ obtained in (19) may be inverted (at least so long as $A$ is a Hilbert-Schmidt operator) to give a generalised normal quantisation of a familiar form. We shall discuss briefly in $\S 6$ how this result may be bettered.

\section{§5. The Algebra $\mathscr{A}$ of Observables for a Compact Semisimple Lie Group $G$}

In the preceding section $H$ and $K$ were trivially identical. This is actually a special case of a more general result.

Theorem 3. Let $M$ be a coadjoint orbit of $G$ in $\mathscr{G}^{*}$ which has a Kähler polarisation, and let $U$ be the representation of $G$ obtained by geometric quantisation. Then the representation space $\mathscr{H}$ of $U$ is a reproducing kernel subspace of the space of $L^{2}$-sections of the canonical line bundle over $M$. If $\Omega$ is the vector corresponding to evaluation at a point $f$ in $M$, then

$$
i \hbar\left\langle\Omega, U_{*}(X) \Omega\right\rangle=f(X) \quad X \in \mathscr{G},
$$

and the stabiliser of the ray through $\Omega$ is the same as the stabiliser of $f$.

Proof. The fact that $\mathscr{H}$ is a reproducing kernel space is shown by Rawnsley [11]. It is also shown there that the reproducing 
kernel defines a symplectic diffeomorphism from $M$ to the projective space of $\mathscr{H}$. Comparison of the moment map of $M$ and on its image in the projective space then leads to $i \hbar\left\langle\Omega, U_{*}(X) \Omega\right)=f(X)$. But since there is a symplectic diffeomorphism from $f$ to the ray through $\Omega$ (which is also a $G$-map), the stabilisers of $f$ and of the ray through $\Omega$ must be identical.

This result provides a valuable tool for interconnecting geometric and normal quantisation. One interesting case in which it applies is the following.

Assume that $G$ is a compact semisimple Lie group and that $\sigma \equiv 1$, so that $\mathscr{H}$ is finite-dimensional. The vectors corresponding to reproducing kernels are the highest weight vectors for maximal tori in $G$. So if $\Omega$ is a highest weight vector the theorem tells us that $H=K$. In this case another simplification occurs.

Theorem 4. If $\Omega$ is a highest weight vector for an irreducble representation of a compact semisimple Lie group $G$, then the map $A \mapsto \phi_{A}$ is an injective map from $\mathscr{B}(\mathscr{H})$ to $L^{2}(G / K)$.

Proof. The kernel of the map $A \mapsto \phi_{A}$ is $\{A \in \mathscr{B}(\mathscr{H}) \mid\langle U(g) \Omega, A U$ (g) $\Omega\rangle=0 \forall g \in G\}$. Introducing the projection $P=|\Omega\rangle\langle\Omega|$ we have that $\langle U(g) \Omega, A U(g) \Omega\rangle=\operatorname{tr}[U(g) P U(g) * A]$, so that $\operatorname{ker} \phi$ is the orthogonal complement in $\mathscr{B}(\mathscr{H})$ of the subrepresentation of $U \otimes U^{*}$ generated by $P$.

Since $\mathscr{H}$ is finite-dimensional, every vector in $\mathscr{H}$ is analytic, and so $U_{*}$ defines a Lie algebra representation of $\mathscr{G}$ by antisymmetric elements of $\mathscr{B}(\mathscr{H})$. In the Lie algebra representation corresponding to $U \otimes U^{*}$ we have

$$
\left[\left(U \otimes U^{*}\right)_{*}(X)\right](B)=\left[U_{*}(X), B\right] \quad X \in \mathscr{G}, B \in \mathscr{B}(\mathscr{H}) .
$$

Now if $X$ is a positive root vector then $U_{*}(X) \Omega=0$ and so

$$
\left[\left(U \otimes U^{*}\right)_{*}(X)\right](P)=-P U_{*}(X) \text {. }
$$

Iterating such operators we see that the subspace generated by $P$ must contain all operators of the form $|\Omega\rangle\langle\xi|$ for any $\xi \in \mathscr{H}$. Using similarly a negative root vector we see that the subspace must contain

$$
U_{*}(X)|\Omega\rangle\langle\xi|-| \Omega\rangle\langle\xi| U_{*}(X) \text { 。 }
$$


Since the second term is already known to belong to the subspace, the subspace must contain all $U_{*}(X)|\Omega\rangle\langle\xi|$ for $X \in \mathscr{G}$ and $\xi \in \mathscr{H}$. Another iterative argument shows that the subspace generated by $P$ contains all operators of the form $|\xi\rangle\langle\eta|$ for $\xi, \eta \in \mathscr{H}$. In other words $P$ generates the whole of $\mathscr{B}(\mathscr{H})$. The kernel of $\phi$ is therefore trivial.

Remark. A similar argument could have been used for the $\sigma$ representation of $\boldsymbol{R}^{2 n}$ considered in the last section, with creation and annihilation operators playing the role of root vectors. However in that case it is simpler to use the direct argument.

For the remainder of this section we shall assume that $G$ is a compact semisimple Lie group, that $U$ is an irreducible representation of $G$, and that $\Omega$ is a highest weight vector. Let us now define $\mathscr{A}$ to be

$$
\mathscr{A}=\left\{\phi_{A} \mid A \in \mathscr{B}(\mathscr{H})\right\}
$$

namely the image of $\mathscr{B}(\mathscr{H})$ under $\phi$. Since $\phi$ is injective it carries the operator product on $\mathscr{B}(\mathscr{H})$ to a product on $\mathscr{A}$ defined by

$$
\phi_{A} * \phi_{B}=\phi_{A B} \quad A, B \in \mathscr{B}(\mathscr{H}) \text {. }
$$

We define $\mathscr{A}_{\circ}$ to be the subspace of $\mathscr{A}$ spanned by the images of the operators $I$ and $i \hbar U_{*}(X)$ for $X \in \mathscr{G}$.

Proposition 5. $\mathscr{A}_{\circ}$ is spanned by the constant functions and the Hamiltonian functions $h(X)$ for $X \in \mathscr{G}$ defined by

$$
[h(X)](m)=m(X) \quad m \in M_{f} \text {. }
$$

Proof. Clearly $\phi_{I}$ is the constant function 1 , and since $[h(X)](g \cdot f)$ $=(g \cdot f)(X)=i \hbar\left\langle U(g) \Omega, U_{*}(X) U(g) \Omega\right\rangle=\phi_{i \hbar U_{*}(X)}(g \cdot f) \quad$ for any $g \in G$, we deduce that $h(X)=\phi_{i \hbar U_{*}(X)}$.

Corollary. $\mathscr{A}_{\circ}$ is a Lie subalgebra of $C^{\infty}\left(M_{f}\right)$ considered as a Lie algebra under the Poisson bracket.

Proof. Since $G$ is semisimple, $h$ is the usual comoment map of geometric quantisation, so that $h([X, Y])=\{h(X), h(Y)\}$ for all $X$, $Y \in \mathscr{G}$. Also we observe that $\left\{1, \phi_{A}\right\}=0$ for all $A \in \mathscr{B}(\mathscr{H})$.

Theorem 5. The product on $\mathscr{A}$ defined by (24) is an $\mathscr{A}_{0}$-invariant 
*-product in the sense of Fronsdal [2].

The proof of this theorem follows immediately from the next lemma.

Lemma. $\phi_{\left[i \hbar U_{*}(X), A\right]}=i \hbar\left\{\phi_{i \hbar U_{*}(X)}, \phi_{A}\right\}$ for all $X \in \mathscr{G}, A \in \mathscr{B}(\mathscr{H})$.

Proof. For any $A \in \mathscr{B}_{S}(\mathscr{H})$, the space of self-adjoint bounded linear maps on $\mathscr{H}$, we observe that $\phi_{A} \in C_{R}^{\infty}\left(M_{f}\right)$, and so we may define the Hamiltonian vector field $\xi_{A}$ on $M_{f}$ by

$$
\xi_{A} \omega+d \phi_{A}=0 \text {. }
$$

Since $M_{f}$ is a transitive $G$-space, for each $m \in M_{f}$ there exists $Z_{m}^{A} \in \mathscr{G}$ such that $\left(\xi_{A}\right)_{m}=Z_{m}^{A}$; in other words

$$
\left(\xi_{A} \phi\right)(m)=\frac{d}{d t} \phi\left(\left.e^{\left.-t Z_{m}^{A} \cdot m\right)}\right|_{t=0} \phi \in C^{\infty}\left(M_{f}\right) .\right.
$$

Thus, for any $Y \in \mathscr{G}$ and $m \in M_{f}$ :

$$
\begin{aligned}
m\left(\left[Y, Z_{m}^{A}\right]\right) & =-\omega_{m}\left(\left(\xi_{A}\right)_{m}, Y\right)=\left(d \phi_{A}\right)_{m}(Y)=\left(Y \phi_{A}\right)(m) \\
& =\left.\frac{d}{d t} \phi_{A}\left(e^{-t Y} \cdot m\right)\right|_{t=0} \\
& =\left.\frac{d}{d t}\left\langle U\left(e^{-t Y}\right) U(g) \Omega, A U\left(e^{-t Y}\right) U(g) \Omega\right\rangle\right|_{t=0} \\
& =\left\langle U(g) \Omega,\left[U_{*}(Y), A\right] U(g) \Omega\right\rangle=\phi_{\left[U_{*}(Y), A\right]}(m)
\end{aligned}
$$

where $m=g \bullet f$ for some $g \in G$. It follows that

$$
m([Y, X])=\phi_{\left.i \hbar U_{*}[Y, X]\right)}(m)=\phi_{i \hbar\left[U_{*}(Y), U_{*}(X)\right]}(m)=m\left(\left[Y, Z_{m}^{i \hbar U_{*}(X)}\right]\right)
$$

for any $X, Y \in \mathscr{G}$ and $m \in M_{f}$, so we deduce that we may safely choose

$$
Z_{m}^{i \hbar U_{*}(X)}=X \quad X \in \mathscr{G}, m \in M_{f} .
$$

The Poisson bracket $\{$,$\} on C_{R}^{\infty}\left(M_{f}\right)$ is defined as usual by $\{\phi$, $\phi\}=\xi_{\phi} \psi$ for $\phi, \phi \in C_{\boldsymbol{R}}^{\infty}\left(M_{f}\right)$, where $\xi_{\phi}$ is the Hamiltonian vector field associated with $\phi$. Thus for any $X \in \mathscr{G}$ we deduce that

$$
\left\{\phi_{i \hbar U_{*}(X)}, \phi_{A}\right\}(m)=\left(Z_{m}^{i \hbar U_{*}(X)} \phi_{A}\right)(m)=\left(X \phi_{A}\right)(m)=\phi_{\left[U_{*}(X), A\right]}(m)
$$

for all $m \in M_{f}$. Hence the result follows if we extend the Poisson bracket structure from $C_{\boldsymbol{R}}^{\infty}\left(M_{f}\right)$ to $C^{\infty}\left(M_{f}\right)$ in the usual complex linear manner.

Remark. By taking $A=i \hbar U_{*}(Y)$ in the Lemma we obtain an 
independent proof that $h([X, Y])=\{h(X), h(Y)\}$.

The proof of Theorem 5 now proceeds by noting that

$$
\begin{gathered}
h(X) * \phi_{A}-\phi_{A} * h(X)=\phi_{\left[i \hbar U_{*}(X), A\right]}=i \hbar\left\{h(X), \phi_{A}\right\} \\
1 * \phi_{A}-\phi_{A} * 1=\phi_{[I, A]}=\phi_{0}=0=i \hbar\left\{1, \phi_{A}\right\}
\end{gathered}
$$

for all $X \in \mathscr{G}, A \in \mathscr{B}(\mathscr{H})$.

Thus we see that the method of normal quantisation is a tool which can be used to calculate Fronsdal *-products. Also, the generalised normal quantisation $\phi_{A} \mapsto A$ provides a representation of the algebra $(\mathscr{A}, *)$, and so automatically quantises (sic) the system.

Finally, we notice that $\mathscr{A}_{\circ}$ is that algebra of observables that we could have quantised had we used the elementary techniques of geometric quantisation. Since $\mathscr{A}_{\circ}$ is generated by the Lie algebra $\mathscr{G}$, it can be interpreted as representing the infinitesimal geometric symmetries of the phase space $M_{f}$, and so is a good candidate for the second algebra in the ${ }^{*}$-product definition of Fronsdal. Since in general the algebra $\mathscr{A}$ will be much larger than $\mathscr{A}_{0}$, the method used here seems to be a great improvement on that of geometric quantisation.

By way of example, consider the representation $D_{m}$ of $S U(2)$, where $2 m \in \mathbf{N}$. The algebra $\mathscr{A}$ is the subspace of $L^{2}(G / H)=L^{2}(S U$ $(2) / U(1))$ which transforms under $S U(2)$ like $D_{m} \otimes D_{m}^{*}=\bigoplus_{j=0}^{2 m} D_{j}$. There is only one such subspace, namely the space of spherical harmonics of order less than or equal to $2 m$. The Lie algebra $\mathscr{A}_{\circ}$, which contains only the zeroth and first order harmonics, will generally be much smaller.

\section{§6. The Group $\mathbb{R}^{2 n}$ (Again)}

The reason that we assumed $G$ compact was so that $\mathscr{H}$ should be finite-dimensional, and hence that every vector $\xi$ in $\mathscr{H}$ should be analytic. We shall now see that we can still achieve this last result (in so far as it is necessary) in this more general case.

We notice that every Schwartz function on $\boldsymbol{R}^{n}$ is analytic for the representation $U$, and that $\Omega \in \mathscr{S}\left(\mathbb{R}^{n}\right)$. Hence the operators $U_{*}(X)$ may be defined on $\mathscr{S}\left(\mathbb{R}^{n}\right)$ for every $X \in \mathscr{G}$, and since 


$$
i \hbar U_{*}\left(J_{k}\right)=-i \hbar \frac{\partial}{\partial q_{k}} i \hbar U_{*}\left(J_{n+k}\right)=-q_{k} \quad 1 \leqq k \leqq n
$$

where $J_{1}, \ldots, J_{2 n}$ is the canonical basis of $\mathscr{G}$, we see that $U_{*}(X) \in$ $\operatorname{End}\left(\mathscr{S}\left(\mathbb{R}^{n}\right)\right)$ for all $X \in \mathscr{G}$.

Since $H=K=\{0\}$, Theorem 1 is trivially true, and we do not need to restrict our attention to bounded linear operators on $L^{2}\left(\mathbb{R}^{n}\right)$. Specifically, if we only consider operators $A$ in $\operatorname{End}\left(\mathscr{S}\left(\mathbb{R}^{n}\right)\right)$, then (19) may be used to define the function $\phi_{A}$ in $\left(C^{\infty}\left(\mathbb{R}^{2 n}\right)\right.$. If we define $\mathscr{E}$ to be the algebra of all elements of End $\left(\mathscr{S}\left(\mathbb{R}^{n}\right)\right)$ which possess adjoints with respect to the natural inner product structure of $\mathscr{S}\left(\mathbb{R}^{n}\right)$, we can construct the linear space

$$
\mathscr{A}=\left\{\phi_{A} \mid A \in \mathscr{E}\right\}
$$

and the subspace

$$
\mathscr{A}_{\circ}=\langle 1, h(X) \mid X \in \mathscr{G}\rangle=\left\langle 1, \xi_{1}, \ldots, \xi_{2 n}\right\rangle .
$$

It is shown in Hennings [5] that the map $\phi: \mathscr{E} \rightarrow \mathscr{A}$ is bijective, and so (24) can be used to define an algebra structure on $\mathscr{A}$. Since every vector that we encounter belongs to $\mathscr{S}\left(\mathbb{R}^{n}\right)$, and every operator belongs to End $\left(\mathscr{S}\left(\mathbb{R}^{n}\right)\right.$ ), we deduce that every vector that we meet in the proof of Theorem 5 is analytic in this case also. Hence the result of Theorem 5 still holds (the proof still works in this case of a projective representation), and so we deduce that $\mathscr{A}_{0}$ is a Lie subalgebra of $C^{\infty}\left(\mathbb{R}^{2 n}\right)$ with respect to the Poisson bracket, and that we

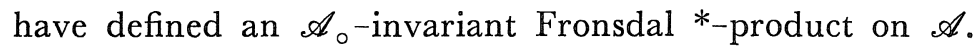

\section{§7. The Boson-Fermion Correspondence}

For an abelian group $G$, provided that $\Phi(g)$ does not vanish we have

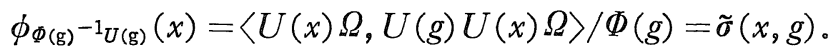

Let us define the subgroup

$$
(G \sigma)=\{g \in G \mid \tilde{\sigma}(x, g)=1 \quad \forall x \in G\} .
$$

It is known that if $\sigma$ is a type I multiplier then the map $g \mapsto \tilde{\sigma}(\circ, g)$ is an isomorphism from $G$ onto $(G \sigma)^{\perp}$, the subgroup of the dual group consisting of characters which are trivial on $(G \sigma)$ (Hannabuss [3]). It follows that when $\sigma$ is a type I multiplier and $\Phi(g)$ does not 
vanish then every element of $(G \sigma)^{\perp}$ can be normal-quantised.

When $\sigma$ is not type $\mathrm{I}$, the map $g \mapsto \tilde{\sigma}(\cdot, g)$ only has an image which is dense in $(G \sigma)^{\perp}$. However, since every character $\chi$ in $(G \sigma)^{\perp}$ can be approximated by a sequence of the form $\tilde{\sigma}\left(\cdot, \gamma_{n}\right)$ one can ask whether $\Phi\left(\gamma_{n}\right)^{-1} U\left(\gamma_{n}\right)$ converges weakly to some operator which provides a normal quantisation $N(\chi)$ of $\chi$. In fact, as the following example shows, it is useful to allow the convergence to take place only on a dense domain, so that $N(\chi)$ can be an unbounded map.

An interesting example of this occurs in the theory of projective repesentations of loop groups, which play an important role in 2dimensional quantum field theory. The following discussion is motivated by Segal [13], and unexplained notation is taken from there.

Let $G=\operatorname{Map}\left(S^{1}, \boldsymbol{T}\right)$ have the multiplier defined by

$$
\sigma\left(e^{i f}, e^{i g}\right)=\exp \left(-\frac{i}{4 \pi}\left(\int_{0}^{2 \pi} g(\theta) f^{\prime}(\theta) d \theta+[f(2 \pi)-f(0)] g(0)\right)\right)
$$

for $f, g \in F_{Z}$. Then we observe that

$$
\tilde{\boldsymbol{\sigma}}\left(e^{i f}, e^{i \mathrm{~g}}\right)=\exp \left(\frac{i}{2 \pi}\left(\int_{0}^{2 \pi} f(\theta) g^{\prime}(\theta) d \theta-\frac{1}{2}[g(2 \pi)+g(0)][f(2 \pi)-f(0)]\right)\right)
$$

and simple calculations show that $(G \sigma)$ is trivial. However $\sigma$ is not of type I because, for instance, for $\zeta \in S^{1}$ the character $\phi \mapsto \frac{\phi(\zeta)}{\phi(1)}$ is not of the form $\phi \mapsto \tilde{\sigma}(\phi, \phi)$ for any $\phi$ in $G$ (unless $\zeta=1$ ). Methods analogous to those of Segal [13] enable us to find a unitary $\sigma$-representation $U$ of $G$.

Let us restrict our attention to the connected component $G_{\circ}$ of the identity of $G$-those functions in $G$ of winding number zero. Then $\left(G_{\circ} \sigma\right)$ is the space of constant functions, so that the characters on $G_{\circ}$ given by $X_{\zeta}(\phi)=\frac{\phi(\zeta)}{\phi(1)}$ and $\chi_{\zeta}(\phi)=\phi(\zeta) \exp \left(-\frac{i}{2 \pi} \int_{0}^{2 \pi} f(\theta) d \theta\right)$ (where $\phi\left(e^{i \theta}\right)=e^{i f(\theta)}$ for $\left.f \in \operatorname{Map}\left(S^{1}, \boldsymbol{R}\right)\right)$ lie in $\left(G_{\circ} \sigma\right)^{\perp}$. If we introduce the function $\phi_{\zeta \lambda}$ in $G_{\circ}$ defined by

$$
\phi_{\zeta \lambda}(z)=\left(1-\lambda \zeta z^{-1}\right)\left(1-\lambda z \zeta^{-1}\right)^{-1} \quad z \in S^{1}
$$

for $0 \leqq \lambda<1$ then

$$
\begin{aligned}
d \log \varphi_{\zeta \lambda} & =\left(\lambda \zeta z^{-2}\left(1-\lambda \zeta z^{-1}\right)^{-1}+\lambda \zeta^{-1}\left(1-\lambda z \zeta^{-1}\right)^{-1}\right) d z \\
& =\left(\sum_{n \neq 0} \lambda^{|n|} \zeta^{n} z^{-(n+1)}\right) d z
\end{aligned}
$$


so if $\phi \in G_{\circ}$, then we can write $\phi=e^{i f}$ where $f(z)=\sum_{n \in Z} f_{n} z^{n}$, and so

$$
\tilde{\boldsymbol{\sigma}}\left(\phi, \phi_{\zeta \lambda}\right)=\exp \left(\frac{1}{2 \pi} \sum_{n \neq 0} \lambda^{|n| \zeta{ }^{n}} \int_{|z|=1} f(z) z^{-(n+1)} d z\right)=\exp \left(i \sum_{n \neq 0} f_{n} \lambda^{|n|} \zeta^{n}\right) .
$$

As $\lambda \rightarrow 1$ we see that

$$
\tilde{\sigma}\left(\phi, \phi_{\zeta \lambda}\right) \rightarrow \exp \left(i \sum_{n \neq 0} f_{n} \zeta^{n}\right)=\chi_{\zeta}(\phi) .
$$

In Segal's notation, if $\zeta=e^{i \alpha}$ then $\phi_{\zeta \lambda}=\gamma_{\alpha 0}^{-1} \circ \gamma_{\alpha \lambda}$, and the argument in [13] shows that $\Phi\left(\psi_{\zeta \lambda}\right)^{-1} U\left(\phi_{\zeta \lambda}\right)$ converges on a dense domain to the operator $U\left(\gamma_{\alpha 0}\right) * B_{\alpha}$, where $B_{\alpha}$ is the (unbounded) fermion operator. Similarly considering $P_{\zeta \lambda}=\gamma_{0 \lambda}^{-1} \cdot \gamma_{\alpha \lambda}$ we can show that $\tilde{\sigma}\left(\phi, P_{\zeta \lambda}\right) \rightarrow X_{\zeta}(\phi)$ as $\lambda \rightarrow 1$, and on the same dense domain as before $\Phi\left(P_{\zeta \lambda}\right)^{-1} U\left(P_{\zeta \lambda}\right) \rightarrow$ $(\zeta-1) B_{0}{ }^{*} B_{\alpha}$ when $\zeta \neq 1$. Thus $U\left(\gamma_{\alpha 0}\right) * B_{\alpha}$ and $(\zeta-1) B_{0} * B_{\alpha}$ are the normal quantisations for $\chi_{\zeta}$ and $X_{\zeta}$ respectively.

One might wonder whether the evaluation character $\phi \mapsto \phi(\zeta)$ on $G$ could be normal-quantised in a similar way. In fact it is easy to check that

$$
\lim _{\lambda \rightarrow 1} \tilde{\sigma}\left(\phi, \gamma_{\alpha \lambda}\right)=\phi(\zeta)
$$

However, the above method does not lead to normal quantisation in this case. This is because $\gamma_{a \lambda}$ has winding number 1 , and so increases the charge of the vacuum vector $\Omega$. Consequently $\Phi\left(\gamma_{\alpha \lambda}\right)$ $=\left\langle\Omega, U\left(\gamma_{\alpha \lambda}\right) \Omega\right\rangle$ vanishes.

\section{References}

[1] Davies, E. B., Quantum theory of open systems, Academic Press (1976).

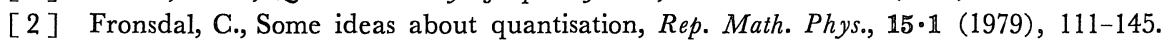

[3] Hannabuss, K. C., Representations of nilpotent locally compact groups, J. Funct. Anal., 34.1 (1979), 146-165.

[4] Some remarks on normal quantisation, Unpublished lecture notes, Oxford (1983).

[5] Hennings, M. A., Fronsdal *-quantisation and the abstract inducing procedures of Fell and Rieffel, D. Phil. thesis, Oxford (1984).

[6] Hudson, R. L., Translation-invariant quantisation and algebraic structures on phase space, Rep. Math. Phys., $10 \cdot \mathbb{1}$ (1976), 9-20.

[7] Husimi, K., Some formal properties of the density matrix, Proc. Phys. Math. Soc. Japan (3), 22 (1940), 264-314.

[8] Kirillov, A. A., Elements of the theory of representations, Springer-Verlag (1976).

[9] Kleppner, A., Multipliers on abelian groups, Math. Ann., 158 (1965), 11-34.

[10] Moyal, J. E., Quantum mechanics as a statistical theory, Math. Proc. Cambridge Philos. Soc., 45 (1949), 99-124. 
[11] Rawnsley, J. H., Coherent states and Kähler manifolds, Quart. J. Math. Oxford (2), 28 (1977), 403-415.

[12] Reiter, H., Über den Satz von Weil-Cartier, Mh. Math., 86 (1978), 13-62.

[13] Segal, G. B., Unitary representations of some infinite-dimensional groups, Commun. Math. Phys., 80 (1981), 301-342.

[14] Woodhouse, N., Geometric quantisation, Clarendon Press (1980). 